\title{
Human and murine PTX1/Ptx1 gene maps to the region for Treacher Collins Syndrome
}

\author{
Michael J. Crawford, ${ }^{1}$ Christian Lanctôt, ${ }^{1}$ Jacques J. Tremblay, ${ }^{1}$ Nancy Jenkins, ${ }^{2}$ Debra Gilbert, ${ }^{2}$ Neal Copeland, ${ }^{2}$ \\ Barbara Beatty, ${ }^{3}$ Jacques Drouin ${ }^{1}$
}

\author{
${ }^{1}$ Laboratoire de génétique moléculaire, Institut de recherches cliniques de Montréal (IRCM), 110 avenue des Pins Ouest, \\ Montréal Québec H2W 1R7, Canada \\ ${ }^{2}$ Mammalian Genetics Laboratory, ABL-Basic Research Program, NCI-Frederick Cancer Research and Development Center, \\ Frederick, Maryland 21702, USA \\ ${ }^{3}$ Hospital for Sick Children, Toronto, Ontario M5G 1X8, Canada
}

Received: 3 May 1997 / Accepted: 1 July 1997

\begin{abstract}
Ptxl belongs to an expanding family of bicoid-related vertebrate homeobox genes. These genes, like their Drosophila homolog, seem to play a role in the development of anterior structures and, in particular, the brain and facies. We report the chromosomal localization of mouse Ptxl, and the cloning, sequencing, and chromosomal localization of the human homolog PTX1. The putative encoded proteins share $100 \%$ homology in the homeodomain and are $88 \%$ and $97 \%$ conserved in the $\mathrm{N}$ - and C-termini respectively. Intron/exon boundaries are also conserved. Murine Ptxl was localized, by interspecific backcrossing, to $\mathrm{Chr} 13$ within $2.6 \mathrm{cM}$ of $\mathrm{Caml}$. The gene resides centrally on Chromosome (Chr) 13 in a region syntenic with human Chr 5q. Subsequent analysis by fluorescent in situ hybridization places the human gene, PTX1, on 5q31, a region associated with Treacher Collins Franceschetti Syndrome. Taken together with the craniofacial expression pattern of Ptxl during early development, the localization of the gene in this chromosomal area is consistent with an involvement in Treacher Collins Franceschetti Syndrome.
\end{abstract}

\section{Introduction}

We recently cloned and characterized a murine transcription factor gene, Ptxl (Lamonerie et al. 1996). The gene was identified on the basis of its ability to activate pituitary transcription of the proopiomelanocortin (POMC) gene. Ptxl is a member of an enlarging family of bicoid-related genes, another member of which, Ptx2/ PTX2 (RIEG), has recently been cloned and identified as the mutant locus underlying Rieger's Syndrome. This syndrome is a developmental anomaly that has its effects primarily in elaboration of the face where the gene is expressed (Semina et al. 1996; Mucchielli et al. 1996; Gage and Camper 1997).

In the developing head, Ptxl is first expressed in the stomodeum. This ectoderm continues to express Ptxl as it gives rise to the epithelia of the oral cavity and first branchial arch and subsequently to their derivatives. These include epithelia of the nasopharynx, palate, tongue, adenohypophysis, olfactory epithelium, submandibular glands, and dental epithelium. Secondarily, expression appears to be induced in a streak of mesenchyme in the first branchial arch: muscle and cartilagenous derivatives of this mesenchyme continue to express Ptxl (Lanctôt et al. 1997). The gene also displays a posterior pattern of expression in the extraembryonic and lateral plate mesoderm; derivatives of the latter include the muscular tissues in the lower body wall, bladder, hindgut, and genital tubercle. Consequently, Ptxl is expressed in the mesen-

Correspondence to: J. Drouin chyme of the hindlimb bud, but never in that of the forelimb (Lanctôt et al. 1997). Given its pattern of expression in the "stomodeal ectomere" (Lanctôt et al. 1997), we wondered if Ptxl might, like PTX2/RIEG, play a role in craniofacial dismorphogenesis: the presentation of Treacher Collins Franceschetti (TCOF) Syndrome is consistent with Ptxl expression patterns and chromosomal localization in the mouse.

TCOF is an autosomal dominant disorder that occurs with a frequency of 1:50,000 live births (Winter 1996; Fazen et al. 1967). The syndrome affects derivatives of the first branchial arch and is characterized by craniofacial abnormalities (Edery et al. 1994). Expressivity and penetrance are variable, and the defects can include combinations of hypoplasia of the malar bones and mandible, conductive hearing loss, malformation of the external ear, enlarged nose, coloboma of the lower lids, and anti-mongoloid slant. In some pedigrees the disorder worsens with successive generations (Fazen et al. 1967), a trait characteristic of anticipation that occurs where mutations commonly involve the expansion of tri- or di- nucleotide repeats (Warren 1996).

Within the last year, a candidate gene (TCOF1) for TCOF has been characterized (Treacher Collins Syndrome Collaborative Group 1996). In some affected patients, the putative protein would suffer from premature truncation owing to insertion, deletion, or aberrant mRNA (Treacher Collins Syndrome Collaborative Group 1996; Gladwin et al. 1997; Wise et al. 1997). However, TCOF1 was mapped to a location that is more proximal than originally anticipated and that indeed may be incompatible with some recombination data (Dixon et al. 1993,1994; Treacher Collins Collaborative Group 1996; Edery et al. 1994). Southern blot analysis of DNA from diverse organisms shows that the locus is not highly conserved. Moreover, TCOF1 is ubiquitously expressed and is highly polymorphic (Treacher Collins Collaborative Group 1996; Wise et al. 1997). None of the TCOF1 mutations was of the type associated with the feature of anticipation displayed in some TCOF pedigrees. TCOF1 may be a pseudogene or merely one of two or more genes involved in the pathogenesis of TCOF. The syndrome also may be heterogenic (Arn et al. 1993). The chromosomal position and developmental pattern of PTX1/Ptxl expression suggest that it may be involved in the pathogenesis of TCOF.

\section{Materials and methods}

Interspecific mouse backcross mapping. Interspecific backcross progeny were generated by mating $(\mathrm{C} 57 \mathrm{BL} / 6 \mathrm{~J} \times M$. spretus $) \mathrm{F}_{1}$ females and C57BL/6J males as described (Copeland and Jenkins 1991). In total, 205 $\mathrm{N}_{2}$ mice were used to map the Ptxl locus (see text for details). DNA isolation, restriction enzyme digestion, agarose gel electrophoresis, South- 
A.
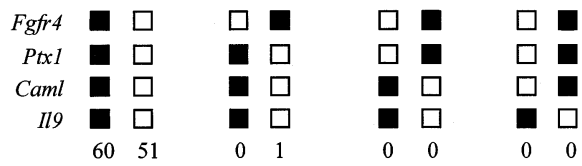

B.

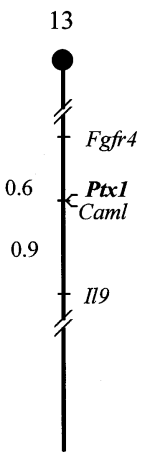

Mouse

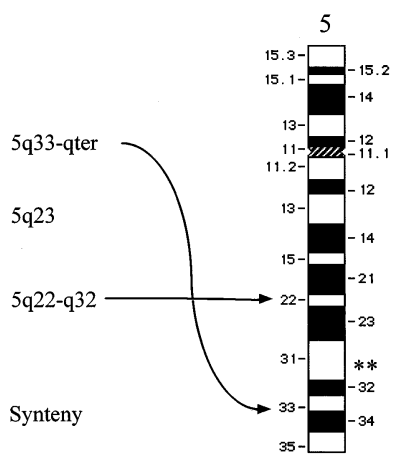

Human
Fig. 1 (A) Ptxl maps in the central region of mouse Chr 13. Ptxl was placed on mouse Chr 13 by interspecific backcross analysis. The segregation patterns of $P t x l$ and flanking genes in 112 backcross animals that were typed for all loci are shown at the top of the figure. For individual pairs of loci, more than 112 animals were typed (see text). Each column represents the chromosome identified in the backcross progeny that was inherited from the $(\mathrm{C} 57 \mathrm{BL} / 6 \mathrm{~J} \times$ M. spretus $) \mathrm{F}_{1}$ parent. The shaded boxes represent the presence of a C57BL/6J allele, and white boxes represent the presence of an M. spretus allele. The number of offspring inheriting each type of chromosome is listed at the bottom of each column. (B) A partial Chr 13 linkage map showing the location of Ptxl in relation to linked genes is shown. Recombination distances between loci in centiMorgans are shown to the left of the chromosome, and the positions of loci in human chromosomes, where known, are shown to the right. References for the human map positions of loci cited in this study can be obtained from GDB (Genome Data Base), a computerized database of human linkage information maintained by The William H. Welch Medical Library of The Johns Hopkins University (Baltimore, Md.). Asterisks indicate the location of fluorescent hybridization signal determined by FISH (Fig. 2.)

ern blot transfer, and hybridization were performed essentially as described (Jenkins et al. 1982). All blots were prepared with Hybond- $\mathrm{N}^{+}$nylon membrane (Amersham). The probe, an 370-bp EcoRI/NcoI fragment of mouse cDNA, was labeled with $\left[\alpha^{32} \mathrm{P}\right] \mathrm{dCTP}$ with a nick translation labeling kit (Boehringer Mannheim); washing was done to a final stringency of $1.0 \times \mathrm{SSCP}\left(20 \times \mathrm{SSCP}\right.$ contains $43.7 \mathrm{~g} \mathrm{Na}_{2} \mathrm{HPO}_{4}, 12.7_{5} \mathrm{NaH}_{5} \mathrm{PO}_{4}$, 88.2g sodium citrate and $140.3 \mathrm{~g} \mathrm{NaCl}$ per liter) $0.1 \% \mathrm{SDS}, 65^{\circ} \mathrm{C}$. A major fragment of $15.0 \mathrm{~kb}$ was detected in HindIII-digested C57BL/6J DNA, and a major fragment of $10.5 \mathrm{~kb}$ was detected in HindIII-digested M. spretus DNA. The presence or absence of the 10.5-kb HindIII M. spretus specific fragment was followed in backcross mice.

A description of the probes and RFLPs for the loci linked to Ptxl, including $\mathrm{Fgfr} 4$, Caml, and $\mathrm{Il} 9$, has been reported previously (Avraham et al. 1994). Recombination distances were calculated as described (Green 1981 ) with the computer program SPRETUS MADNESS. Gene order was determined by minimizing the number of recombination events required to explain the allele distribution patterns.

Isolation of human PTX1 and FISH mapping. Sequences from murine Ptxl cDNA were employed to amplify fragments of cDNA from reverse-transcribed human pituitary tumor mRNA (forward primer TCTCTTGTCCCCACAGGTC, reverse primer CCCAGTTGTTGTAGGAGTAGCC). The 639-bp fragment derived in this manner was used as a probe to screen a genomic library constructed in XZAP. In a screen of $2 \times$ $10^{5}$ plaque-forming units, one positive clone was isolated that contained only the first two exons of the gene. The missing $3^{\prime}$ exon was subcloned from a PAC (courtesy Canadian Genome Analysis and Technology program, Canada) that was isolated with sequences from the $3^{\prime}$ end of the genomic clone and the cDNA fragment as probes. Double-stranded DNA sequencing was accomplished with a chain termination protocol (Sanger et al. 1977).

Fluorescent in situ hybridization (FISH) was performed on normal human lymphocyte chromosomes (Lichter et al. 1990). The probe employed was a biotinylated 13-kb genomic fragment of human PTX1 that was detected with avidin-fluorescein isothiocyanate (FITC). Counterstaining was done with propidium iodide and 4',6-diamidine-2-phenylindoldihydrochloride (DAPI; Heng and Tsui 1993). Separate DAPI and FITC images of metaphase spreads were recorded through a thermoelectrically cooled charge coupled camera (Photometrics, Tuscon, Ariz.) and superimposed digitally (Boyle et al. 1992). Band assignment was determined by measuring the fractional chromosome length and by comparison with the DAPI banding pattern (Heng and Tsui 1993).

The structure of the PTX1 locus was determined by comparison of genomic with cDNA sequences as well as with their murine and chick counterparts. The murine gene structure was determined by mapping genomic restriction fragments with cDNA probes, sequence analysis, and PCR amplification. The genomic clone was also examined for the presence of trinucleotide repeats with oligonucleotides as probe; namely, $(\mathrm{CCG})^{6}$, $(\mathrm{CTG})^{6}$, and (GAA) ${ }^{6}$. Oligonucleotides were labeled by use of terminal transferase and ${ }^{32} \mathrm{P}$-dCTP. The human PTX1 sequence was submitted to Genbank (accession numbers AF009648, AF009649, AF009650).

\section{Results}

The mouse chromosomal location of Ptxl was determined by interspecific backcross analysis. This interspecific backcross map-
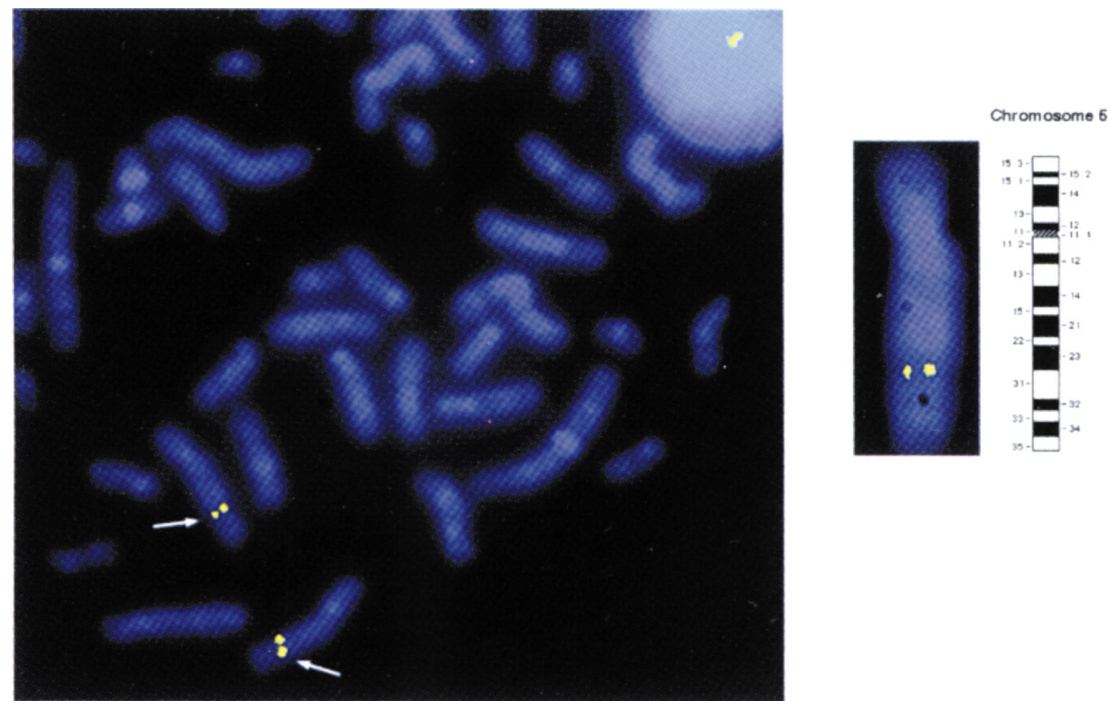

Fig. 2. Fluorescent in situ hybridization links PTX1 to $\mathrm{Chr} 5 \mathrm{q} 31$. Paired copies of PTX1 are detectable on Chr 5 in human lymphocyte metaphase spreads. Arrows indicate sites of hybridization with PTX1 genomic fragments. Positional assignment was made on the basis of fractional chromosome length and DAPI banding patterns. 
A.

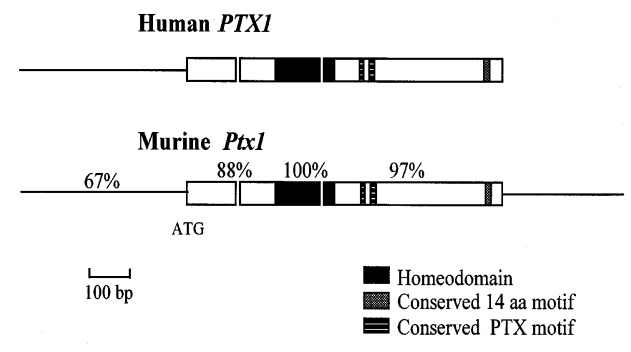

B.

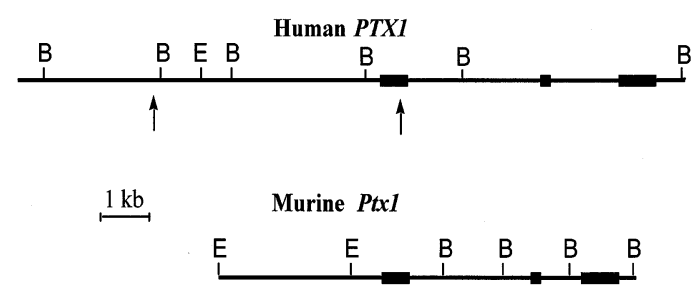

C.

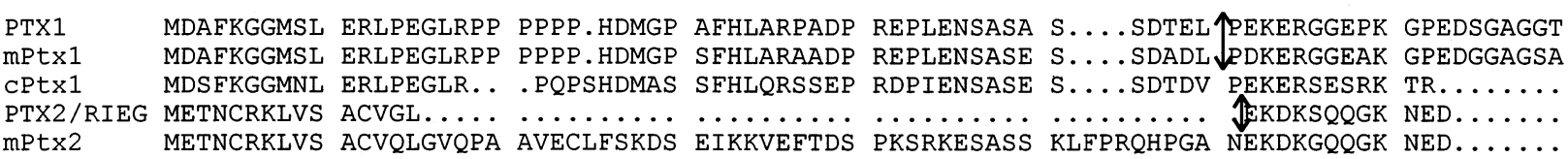

PTX1 GCGGGADDPA KKKKপRRQRT HFTSQQLQEL EATFQRNRYP DMSMREEIAV WTNLTEPRVR个VWFKNRRAKW RKRERNQQLD mPtx1 GCGGGAEDPA KKKKØRRQRT HFTSQQLQEL EATFQRNRYP DMSMREEIAV WTNLTEPRVR VWFKNRRAKW RKRERNQQLD CPTX1 ‥TGAADDPA KKKKØRRQPT HFTSQQLQEL EATFQRNRYP DMSMREEIAV WTNLTEPRVR VWFKNRRAKW RKRERNQQMD PTX2/RIEG …VGAEDPS KKKR RRQRT HFTSQQLQEL EATFQRNRYP DMSTREEIAV WTNLTEARVR IVWFKNRRAKW RKRERNQQAE mPtx2 ‥VGAEDPS KKKR RRRORT HFTSOOLOEL EATFORNRYP DMSTREEIAV WTNLTEARVR VWFKNRRAKW RKRERNQQAE

PTX1 LCKGGYVPQF SGLVQPYEDV YAAGYSYNNW AAKSLAPAPL STKSFTFFNS M..SPLSSQS MFSAPSSISS MTMPSSMGPG mPtX1 LCKGGYVPQF SGLVQPYEDV YAAGYSYNNW AAKSLAPAPL STKSFTFFNS M..SPLSSQS MFSAPSSISS MTMPSSMGPG CPtX1 LCKNGYVPQF SGLMQPYDDM Y.AGYPYNNW ATKSLTPAPL STKSFTFFNS M..SPLSSQS MFSAPSSISS MNMPSGMGHS PTX2/RIEG LCKNGFGPQF NGLMQPYDDM Y.PGYSYNNW AAKGLTSASL STKSFPFFNS M.VNPLSSQS MFSPPNSISS MSMSSSMVPS mPtX2 LCKNGFGPQF NGLMQPYDDM Y.PGYSYNNW AAKGLTSASL STKSFPFFNS MNVNPLSSQS MFSPPNSISS MSMSSSMVPS PTX Family Motifs

PTX1 AVPGMPNS.. GLNNINNLT GSSLNSAM.S PGACPYGTPA SPYSVYRDTC NSSLASLRLK SKQHSSFGYG GLQGPASGLN mPtx1 AVPGMPNS.. $\quad$ GLNNINNLT GSSLNSAM.S PGACPYGTPA SPYSVYRDTC NSSLASLRLK SKQHSSFGYG GLQGPASGLN CPtX1 AVPGMANS.. .GLNNINNIS GSSLNSAMSS P.ACPYGPPG SPYSVYRDTC NSSLASLRLK SKQHSSFGYS SLQSPGSSLN PTX2/RIEG AVTGVPGSSL NSLNNLNNLS \$SLNSAVPT P.ACPTAPPT PPT.VTRDTC NSSLASLRLK AKQHSSFGYA SVQKPASNLS mPtx2 AVTGVPGSSL NSLNNLNNLS SPSLNSAVPT P.ACPYAPPT PPY.VYRDTC NSSLASLRLK AKQHSSFGYA SVQNPASNLS

$\begin{array}{ll}\text { PTX1 } & \text { ACQYNS } \ldots \\ \text { mPtX1 } & \text { ACQYNS.... } \\ \text { CPtX1 } & \text { ACQYNS } \ldots \\ \text { PTX2/RIEG } & \text { ACQTAVDRPV } \\ \text { mPtX2 } & \text { ACQYAVDRPV }\end{array}$

ping panel has been typed for over 2300 loci that are well distributed among all the autosomes as well as the $\mathrm{X} \mathrm{Chr} \mathrm{(Copeland} \mathrm{and}$ Jenkins 1991). The mapping results indicated that Ptxl is located in the central region of mouse Chr 13 linked to Fgfr4, Caml, and Il9. Although 112 mice were analyzed for every marker and are shown in the segregation analysis (Fig. 1a), up to 179 mice were typed for some pairs of markers. Each locus was analyzed in pairwise combinations for recombination frequencies with the additional data. The ratios of the total number of mice exhibiting recombinant chromosomes to the total number of mice analyzed for each pair of loci and the most likely gene order are: centromere-Fgfr4-1/179-Ptxl-0/115-Caml-1/115-Il9. The recombination frequencies [expressed as genetic distances in centiMorgans
(cM) \pm the standard error] are $-F g f r 4-0.6 \pm 0.6-[$ Ptxl, Camfl-0.9 $\pm 0.9-I l 9]$. No recombinants were detected between Ptxl and Caml in 115 animals typed in common, suggesting that the two loci are within $2.6 \mathrm{cM}$ of each other (upper $95 \%$ confidence limit). Ptxl mapped in a region of the composite map that lacks mouse mutations with a phenotype that might be expected for an alteration in this locus (data not shown); however, an extinct mouse mutation, dumpy (dpy), that affects development of the snout and hindlimbs was localized to Chr 13 (Hollander 1981). The central region of mouse Chr 13 shares a region of homology with human $\mathrm{Chr} 5 \mathrm{q}$ (Fig. 1b). In particular, Caml has been placed near human 5q23. The tight linkage between Ptxl and Caml in mouse suggested that Ptxl resides on 5q, as well. 
A 13-kb human genomic probe was used to identify the PTX1 locus on chromosomal spreads. FITC-labeled probe was localized to $5 \mathrm{q} 31$ by analyzing the DAPI banding patterns and measuring the fractional chromosomal length. Analysis of 20 metaphase spreads demonstrated that positive fluorescent in situ hybridization signals were observed in 14 out of 20 spreads, and signals could be identified on both homologs in 9 of these (Fig. 2.). The chromosomal localization of PTX1 places the gene proximal to TCOF1 but within the region of critical interest for TCOF (Edery et al. 1994; Dixon et al. 1994; Treacher Collins Syndrome Collaborative Group, 1996).

PTX1, like its murine and chick homologs, possesses a homeodomain with a bicoid-class third helix. The homeodomain is highly conserved between mouse and human (100\%), as are the Cand $\mathrm{N}$ - termini ( 88 and $97 \%$ respectively; Fig. 3a,b). This conservation is also evident at the level of gene structure as the murine and human genes both have similar organization: the intron/exon borders are identical. Human PTX1 is also conserved when compared with sequences obtained from chick (Fig. 3c). Like products of the PTX2 gene family, the PTX1 homologs encode a conserved C-terminal sequence of 14 residues noted to be present in other homeobox genes that share periods of expression in craniofacial structures during development (Semina et al. 1996). Moreover, there appear to be two short consensus sequences just C-terminal of the homeodomain that are present in members of the PTX family. Finally, a trinucleotide repeat $(\mathrm{GCC})^{5}$ is present in the open reading frame of the first exon. Similar repeats are also present upstream of the gene. We have not yet identified signs of repeat expansion in TCOF probands; however, genomic Southern blots have not always proven reliable when repeat expansion alleles are chimeric in length and representation. In summary, the fine mapping of murine Ptxl and human PTX1 indicates conservation in sequence, organization, and chromosomal localization on syntenic regions of mouse and human Chr 13 and 5 respectively.

\section{Discussion}

Taken together with the localization of the PTX1 gene in the area critical for TCOF, the murine pattern of Ptxl expression in the stomodeum and its derivatives is highly suggestive of an involvement of PTX1 in the clinical manifestations of TCOF. PTX1 and TCOF1 are close on Chr 5 and would probably cosegregate: mutations in the PTX1 locus might account for TCOF in families that did not exhibit TCOF1/Treacle mutations, or it might itself be the causative gene if TCOF1 is a pseudogene. Further, the ubiquitously expressed TCOF1/Treacle gene product, it if exists, must mediate its effect through another agent that exhibits tissue specificity, like Ptxl. PTX1 is a member of the same gene subfamily that encodes PTX2 (RIEG) and displays a similar yet distinct expression pattern consistent with a role in facial development (Semina et al. 1996; Mucchielli et al. 1996; Lanctôt et al. 1997). TCOF, like Rieger's Syndrome, is autosomal dominant and could also exert its effect through haploinsufficiency. Finally, the localization of PTX1 expression to the pituitary anlage and organ is not inconsistent with an involvement in TCOF because PTX2 (RIEG) has a similar period of activity in Rathke's pouch. Neither Rieger's nor TCOF is usually associated with endocrine symptoms: PTX1 and PTX2 may be redundant in the pituitary, whereas their differences in craniofacial mesenchyme expression may account for the differences between the two syndromes.

Sporadic references to ancillary anomalies appear throughout the TCOF literature (Hunt and Smith 1955; Rogers 1964; Szlazak 1953), and these anomalies occur in regions where Ptxl is transcribed in mouse. For example, some TCOF patients have abnormalities of dentition, feet, digestive, and reproductive tracts that are all domains of early murine Ptxl expression. Furthermore, abnormal expression or function of PTX1 in the foregut endoderm may result in posterior displacement of the soft palate and epiglottis during buccal/pharyngeal fusion in TCOF patients.

We are currently assessing TCOF families for the presence of mutations in the PTX1 locus.

Acknowledgments. We thank Drs. P. Gage and S. Camper, and P. Burbach for communication of results prior to publication, and also to A. Marcil, Y. Gauthier, M. Chamberland, and B. Householder for excellent technical assistance. Thanks are also due to Drs. S. Scherer and J. Huizenga of the CGAT Physical Mapping Resource Facility; and Z.M. Zhang and T. Scheidl of the CGAT FISH Mapping Centre for excellent technical support. This research was supported, in part, by the National Cancer Institute, DHHS, under contract with ABL to N. Copeland and N. Jenkins, a grant from the National Cancer Institute of Canada supported with funds provided by the Canadian Cancer Society to J. Drouin, and the Canadian Genome Analysis and Technology program.

\section{References}

Arn PH, Mankinen C, Jabs EW (1993) Mild mandibulofacial dysostosis in a child with a deletion of 3p. Am J Med Genet 46, 534-536

Avraham KB, Givol D, Avivi A, Yayon A, Copeland NG, Jenkins NA (1994) Mapping of murine fibroblast growth factor receptors refines regions of homology between mouse and human chromosomes. Genomics $21,656-658$

Boyle AL, Feltquite DM, Dracopoli NC, Housman DE, Ward DC (1992) Rapid physical mapping of cloned DNA on banded mouse chromosomes by fluorescence in situ hybridization. Genomics 12 , 106-115

Copeland NG, Jenkins NA (1991) Development and applications of a molecular genetic linkage map of the mouse genome. Trends Genet 7 , $113-118$

Dixon J, Gladwin AJ, Loftus SK, Riley JH, Perveen R, Wasmuth JJ, Anand R, Dixon MJ (1994) A YAC contig encompassing the Treacher Collins syndrome critical region at 5q31.3-32. Am J Hum Genet 55, 372-378

Dixon MJ, Dixon J, Houseal T, Bhatt M, Ward DC, Klinger K, Landes GM (1993) Narrowing the position of the Treacher Collins syndrome locus to a small interval between three new microsatellite markers at 5q32-33.1. Am J Hum Genet 52, 907-914

Edery P, Manach Y, Le Merrer M, Till M, Vignal A, Lyonnet S, Munnich A (1994) Apparent genetic homogeneity of the Treacher CollinsFranceschetti syndrome. Am J Med Genet 52, 174-177

Fazen LE, Elmore J, Nadler HL (1967) Mandibulo-facial dysostosis. (Treacher-Collins syndrome). Am J Dis Child 113, 405-410

Gage PJ, Camper SA (1997) Pituitary homeobox 2, a novel member of the bicoid-related family of homeobox genes, is a potential regulator of anterior structure formation. Hum Mol Genet 6, 457-464

Gladwin AJ, Dixon J, Loftus SK, Edwards S, Wasmuth JJ, Hennekam RCM, Dixon MJ (1997) Treacher Collins Syndrome may result from insertions, deletions or splicing mutations, which introduce a termination codon into the gene. Hum Mol Genet 5, 1533-1538

Green EL (1981) Linkage, Recombination and Mapping. (New York: Oxford University Press), pp 77-113

Heng HH, Tsui LC (1993) Modes of DAPI banding and simultaneous in situ hybridization. Chromosoma 102, 325-332

Hollander WF (1981) Linkage relations of dumpy, a recessive mutant on chromosome 13 of the mouse. J Hered 72, 358-359

Hunt PA, Smith DI (1955) Mandibulo-facial dysostosis. Pediatrics 15, 195-199

Jenkins NA, Copeland NG, Taylor BA, Lee BK (1982) Organization, distribution, and stability of endogenous ecotropic murine leukemia virus DNA sequences in chromosomes of Mus musculus. J Virol 43, 26-36

Lamonerie T, Tremblay JJ, Lanctôt C, Therrien M, Gauthier Y, Drouin J (1996) PTX1, a bicoid-related homeobox transcription factor involved in transcription of pro-opiomelanocortin (POMC) gene. Genes Dev 10, $1284-1295$

Lanctôt C, Lamolet B, Drouin J (1997) The bicoid-related homeoprotein Ptxl defines the most anterior domain of the embryo and differentiates posterior from anterior lateral mesoderm. Development 124, 2807-2817

Lichter P, Tang CJ, Call K, Hermanson G, Evans GA, Housman D, Ward DC (1990) High-resolution mapping of human chromosome 11 by in situ hybridization with cosmid clones. Science 247, 64-69

Mucchielli ML, Martinez S, Pattyn A, Goridis C, Brunet JF (1996) Ottx2, and $O t x$-related homeobox gene expressed in the pituitary gland and in a restricted pattern in the forebrain. Mol Cell Biol 8, 258-271 
Rogers B (1964) Berry-Treacher Collins syndrome: a review of 200 cases. Br J Plast Surg 17, 109-136

Sanger F, Nicklen S, Coulson AR (1977) DNA sequencing with chainterminating inhibitors. Proc Natl Acad Sci USA 74, 5463-5467

Semina EV, Reiter R, Leysens NJ, Alward WL, Small KW, Datson NA, Siegel-Bartelt J, Bierke-Nelson D, Bitoun P, Zabel BU, Carey JC, Murray JC (1996) Cloning and characterization of a novel bicoid-related homeobox transcription factor gene, RIEG, involved in Rieger syndrome. Nature Genet 14, 392-399

Szlazak J (1953) Treacher Collins syndrome. Can Med Assoc J 69, $274-$ 276
Treacher Collins Syndrome Collaborative Group (1996) Positional cloning of a gene involved in the pathogenesis of Treacher Collins syndrome. Nature Genet 12, 130-136

Warren ST (1996) The expanding world of trinucleotide repeats. Science 271, 1374-1375

Winter RM (1996) What's in a face? Nature Genet 12, 124-129

Wise CA, Chiang LC, Paznekas WA, Sharma M, Musy MM, Ashley JA, Lovett M, Jabs EW (1997) TCOF1 gene encodes a putative nucleolar phosphoprotein that exhibits mutations in Treacher Collins Syndrome throughout its coding region. Proc Natl Acad Sci USA 94, 3110-3115 